\title{
Consumption of Brazil Nuts Provides Cardiovascular Health Benefits
}

\author{
Mariana Sarto Figueiredo \\ Universidade Federal Fluminense, Niterói, RJ - Brazil
}

\section{Indroduction}

The prevalence of chronic diseases, such as metabolic syndrome, obesity, dyslipidemia, type 2 diabetes mellitus (DM2), cancer, and neurodegenerative and cardiovascular disorders have been increasing in the world population. However, cardiovascular diseases remain the leading cause of mortality and morbidity worldwide. According to the Barker's Theory (1993), hormonal, nutritional or environmental changes during critical periods of development such as gestation, lactation or adolescence may result in changes to the basic functions of the human body in childhood and "program" the progeny for the emergence of chronic diseases in the adult life, like DM2, obesity, cardiovascular diseases, intestinal dysbiosis, and hormonal and metabolic dysregulation. This phenomenon is known as "metabolic programming" or "developmental plasticity". 1,2

In the same direction, many authors have demonstrated the impact of an unhealthy diet on the development of chronic diseases such as metabolic syndrome, obesity, dyslipidemia, type 2 diabetes mellitus, cancer, neurodegenerative diseases and increased cardiovascular disorders. ${ }^{3,4}$ On the other hand, we know that the regular consumption of plant-based foods (cereals, fruits, vegetables, legumes, tree nuts and seeds), moderate consumption of seafood, fish and dairy and reduced consumption of alcohol, red meat and meat products may prevent and/or protect against diseases and provide health benefits, which is now widely recognized by health professionals. ${ }^{5,6}$

\section{Keywords}

Nuts; Brazil Nuts; Cardiovascular Diseases; Risk Reduction Behavior; Healthy Diet; anti-Inflammatory Agents.
Nutrition is essential for life and essential chemical compounds from foods and drinks such as proteins, fatty acids, carbohydrates, vitamins and minerals are required by the organism to support its physiological functions, like energy production, growth, development and reproduction. ${ }^{7}$ Thereby, nutrients are one of the most important elements that can regulate enzymes and molecular and functional events in the cells or in the whole body, which, depending on the quality and quantity, can predispose humans to chronic diseases. ${ }^{8}$ It is important to highlight that, particularly after the Industrial Revolution worldwide, the diet of modern human society is actually based on processed foods, , which are rich in sugar, salt and saturated fat and poor in minerals and vitamins.

Thus, the inclusion of food with functional properties or bioactive compounds such as monounsaturated fatty acids (MUFA) and polyunsaturated fatty acids (PUFA), phenolic compounds and minerals are gaining increasing recognition as integral components of lifestyle changes against the development of cardiovascular diseases. Recently, the review that presents the association of nuts with cardiovascular diseases highlights some of these compounds present in food that can add important benefits to the cardiovascular health. ${ }^{9}$ In this direction, the nuts (Brazil nuts, American almonds, pistachios, walnuts and hazelnuts) are an important source of MUFA and PUFA, which present anti-inflammatory and antioxidant effects, improve dyslipidemia and contain selenium and phenolic acids. These compounds could also benefit the cardiovascular health.

Silva et al. (2018) showed that regular consumption of 5 to 50 grams of Brazil nuts from 1 day to 16 weeks can increase plasma selenium, improve oxidative balance (increase GPx activity and nitric oxide with decreased MDA), improve lipid profile (increase HDL-c, decrease LDL-c and triglycerides) and reduce inflammation

Mailing Address: Mariana Sarto Figueiredo

Universidade Federal Fluminense - Nutrition and Dietetic - Rua Mário Santos Braga, n 30, 4 andar. Postal Code: 24220-900, Valonguinho, Centro , Niterói, RJ - Brazil.

E-mail: marisartof@gmail.com 
markers (decrease IL-6, TNF-alfa and Nf-kB) in different human populations such as adults, normolipidemic subjects, healthy volunteers, obese adolescents and women, as well as dialysis, hypertensive and dyslipidemic patients. ${ }^{9}$ Silva et al. ${ }^{9}$ did not only revise the effects of nuts on cardiovascular diseases but also highlighted Brazil nuts in terms of their source of unsaturated fatty acids, proteins, fibers, minerals, vitamins, phenolic compounds and properties, biological effects, and proposed mechanisms of action. ${ }^{9}$ They also discussed promising research directions for the future to identify additional health-related benefits of dietary Brazil nuts against cardiovascular diseases.

Recently, Garcia-Aloy et al., ${ }^{10}$ demonstrated that some nuts and vegetables oils are sources of fatty acids, micronutrients and phytochemicals that can be found in blood circulation and in urine according to their intake, and useful to determine habitual intake of nuts as wells as their derived metabolites. ${ }^{10}$ Thus, it is important to evaluate the specificity, sensitivity, dose-response relationships, and determine the relationship with cardiovascular diseases.

In conclusion, it is known that dietary intervention based on the use of foods with functional properties, especially Brazil nuts, can be considered a good strategy to prevent, treat or reduce the progression of cardiovascular diseases worldwide. In my opinion, more studies are necessary to elucidate which cellular mechanisms are involved in the nutritional route of the body metabolism and gene expression of this bioactive compounds and its impact on the cardiovascular health.

\section{References}

1. Barker DJ, Martyn CN, Osmond C, Hales CN, Fall CH. Growth in utero and serum cholesterol concentrations in adult life. BMJ. 1993,307(6918):1524-7.

2. Li X, Zhang M, Pan X, Xu Z, Sun M. “Three Hits" hypothesis for developmental origins of health and diseases in view of cardiovascular abnormalities. Birth Defects Res. 2017;109(10):744-57.

3. Fardet A, Boirie Y. Associations between food and beverage groups and major diet-related chronicdiseases: an exhaustive review of pooled/ meta-analyses and systematic reviews. Nutr Rev. 2014;72(12):741-62.

4. Quintanilha BJ, Reis BZ, Duarte GBS, Cozzolino SMF, Rogero MM. Nutrimiromics: role of microRNAs and nutrition in modulating inflammation and chronic diseases. Nutrients. 2017;9(11):pii:E1168.

5. Lăcătușu CM, Grigorescu ED, Floria M, Onofriescu A, Mihai BM. The Mediterranean Diet: from an environment-driven food culture to an emerging medical prescription. Int J Environ Res Public Health. 2019;16(6):pii:E942.

6. Bruins MJ, Van Dael P, Eggersdorfer M. The role of nutrients in reducing the risk for noncommunicable diseases during aging. Nutrients. 2019;11(1):pii:E85.

7. Jacobs DR Jr. What comes first: the food or the nutrient? Executive summary of a symposium, J Nutr. 2014;144(4 Suppl):543S-546S.

8. Boaz NT. Evolving health: the origins of illness and how the modern world is making us sick. New York: Wiley \& Sons; 2002.

9. Silva ACT, Cardozo LFMF, Cruz BO, Mafra D, Stockler-Pinto MB. Nuts and cardiovascular diseases: focus on Brazil nuts. Int J Cardiovasc Sci. 2019 Apr 01; [Epub ahead of print].

10. Garcia-Aloy M, Hulshof PJM, Estruel-Amades S, Osté MCJ, Lankinen M, Geleijnse JM, et al. Biomarkers of food intake for nuts and vegetable oils: an extensive literature search. Genes Nutr. 2019;14(7):1-21. 